\title{
Ascorbic acid promotes 3T3-L1 cells adipogenesis by attenuating ERK signaling to upregulate the collagen VI
}

\author{
Chuanguo Liu, Kun Huang, Guorong Li, Pingping Wang, Chang Liu, Congcong Guo, Zongguo Sun and Jie Pan*
}

\begin{abstract}
Background: Type $\mathrm{VI}$ collagen is supposed to be a regulation factor in adipogenesis. This study aimed to assess the promoting effect of vitamin C (VC) on adipogenic differentiation of preadipocytes as well as its mechanism.

Methods: Five sets of different combinations of chemicals were used to inhibit synthesis of type I to VI collagens, blocking ERK1/2 phosphorylation during adipogenesis of 3T3-L1 preadipocytes. Furthermore, to explore whether collagen VI plays a critical role during adipogenesis, specific knockdown of collagen VI was performed by using RNA interference. The morphology and expression patterns of several target factors involved in adipogenesis were assessed at various time points.

Results: A reduction in ERK1/2 phosphorylation and an increase in collagen $\mathrm{VI}$ and adipogenic-specific factors, such as C/EBP $\beta$, PPARY and C/EBPa, were observed after treating adipogenic 3T3-L1 cells with AA2P, a stable derivative of VC. Inhibition of collagen synthesis by ethyl-3, 4-dihydroxybenzoate (EDHB) or by specific knockdown of collagen VI by RNAi could promote ERK1/2 phosphorylation. The ERK1/2 phosphorylation in both cases could be attenuated by AA2P treatment. In addition, the inhibition of ERK1/2 phosphorylation by U0126, a highly selective inhibitor of both MEK1 and MEK2 and a type of MAPK/ERK kinase, up-regulated the expression of collagen VI, while it downregulated the adipogenic-specific factors.
\end{abstract}

Conclusion: AA2P could up-regulate the expression of collagen VI by attenuating ERK1/2 phosphorylation, further up-regulating adipocyte-specific factors, thus finally promoting the adipogenesis of 3T3-L1 preadipocytes.

Keywords: Ascorbic acid, 3T3-L1 cells, Collagen VI, ERK signaling, Adipogenesis

\section{Background}

In addition to significant increases in protein expression directly related to lipid metabolism, the type and level of ECM components secreted during adipogenesis also change $[1,2]$. It has been reported that the progression from preadipocytes to mature adipocytes is required for remodeling of the ECM [3]. Collagens in the ECM are secreted with the differentiation of adipocytes, and each type of collagen secretes at a specific stage of adipogenesis. Collagens I and III are secreted in the early stage of adipogenesis to construct the cell scaffolds. Basal membrane components, such as collagen IV, reached a peak in the middle stage of adipogenesis. Microfiber collagen

\footnotetext{
* Correspondence: jiepan@sdnu.edu.cn

Shandong Provincial Key Laboratory of Animal Resistant Biology, College of Life Sciences, Shandong Normal University, Jinan 250014, China
}

components such as collagen V and VI secretion also reached a maximum level during adipogenesis [4]. This remodeling, which encompasses increased flexibility of ECM and changes in the connection among collagens, can promote the transcription of adipocyte-specific genes, such as CCAAT/enhancer-binding protein $\beta(\mathrm{C} /$ EBP $\beta$ ) and peroxisome proliferator-activated receptor $\gamma$ (PPAR $\gamma$ ) [5].

Collagen VI is comprises the three subunits of $\alpha 1, \alpha 2$ and $\alpha 3$, which are encoded by different genes. The silencing of the Col6 $\alpha 1$ gene can prevent the secretion and assembly of collagen VI in the ECM, affecting its function [6-8]. Compared to other types of collagen, collagen VI is enriched in adipocytes and is associated with adipogenesis and obesity-related diseases $[1,2,9,10]$. However, the roles played by collagen VI in regulating 
adipogenesis or adipocyte hypertrophy have not been fully addressed.

Ethyl-3, 4-dihydroxybenzoate (EDHB) is a known ideal inhibitor through which we can explore the role of collagens in regulating adipogenesis of preadipocytes [1]. It inhibits the activity of prolyl-4-hydroxylase, resulting in a non-functional degradable protein and the failure of collagen triple helix formation and preventing the secretion and assembly of type I-VI collagens [11]. It has been reported that collagen $\mathrm{V}$ and $\mathrm{VI}$ play a primary role in the adipogenesis of the bovine intramuscular preadipocyte cell line (BIP). The triglyceride (TG) content of BIP is reduced by $50 \%$ after EDHB treatment. Moreover, the adipogenic rate restores $23.2 \%$ and $31.5 \%$ of the cells cultured on dishes coated with collagen V and VI, respectively. There was no significant effect of BIP on TG accumulation, which was cultured on dishes coated with other types of collagen [1]. These data indicated that collagen VI plays a primary role during adipogenesis, while lacking a discussion of its mechanism.

In addition to its antioxidant activity, vitamin $\mathrm{C}$ (VC) acts as a cofactor of the hydroxylating enzyme of proline and lysine residues in procollagen, which stabilizes the collagen triple helix. Moreover, VC regulates collagen synthesis, cell growth, and cell differentiation [12, 13]. It has been reported that VC can enhance stem cells to differentiate into cardiac lineages by enhancing collagen synthesis [14-16], and it can enhance neurons by increasing the expression of genes involved in neurogenesis [17]. In addition, AA2P, a stable derivative of VC that possesses similar biological properties has been demonstrated to stimulate lipid accumulation by increasing the collagen IV synthesis [18] and through differential expression of collagens [19]. Moreover, AA2P also promotes adipogenesis of BMSCs by increasing the accumulation of collagens, but it does not specify the type of collagen [20, 21]. Therefore, the question remains as to whether differential expression of collagens and the accumulation of collagen stimulate lipid accumulation.

Extracellular signal-regulated kinase (ERK) is a member of the MAPK family, which controls cell proliferation, differentiation and stress response [22]. The ERK signal plays an important role in adipogenesis; however, its specific effect is divergent. In fact, several studies suggest that ERK1/2 could promote adipogenesis and U0126, which is a specific inhibitor of MEK1 and MEK2; thus, ERK inactivation could inhibit adipogenesis [23-25]. While others propose an inhibitory role for ERK1/2 during adipogenesis [26, 27].

There is also a link between VC and ERK. It has been reported that AA2P promotes the production of ECM components by attenuating the phosphorylation of ERK1/2 and enhances the stemness of adipose-derived stem cells and the cell sheet formation, while it has no effect on the subsequent adipogenic differentiation [28]. In other studies, $\mathrm{VC}$ attenuates the phosphorylation of ERK1/2 to inhibit the regulation of type I and III collagen production by the LL-37 polypeptide in human dermal fibroblasts [29]. Based on the above evidence, we hypothesized that AA2P attenuates the phosphorylation of ERK1/2 to up-regulate the expression of collagen VI and promote the adipogenesis of 3T3-L1 cells.

\section{Methods \\ Chemicals}

AA2P, EDHB and U0126 were purchased from SigmaAldrich (Milwaukee, Wisc., USA). AA2P was initially dissolved in Dulbecco's modified Eagle's medium (DMEM, Hyclone) in a $250 \mathrm{mM}$. EDHB was initially dissolved in absolute ethanol in a $100 \mathrm{mM}$. U0126 was initially dissolved in dimethyl sulfoxidein (DMSO) in a $100 \mathrm{mM}$. The final ethanol and DMSO concentration of the controls were adjusted to $0.1 \%$.

\section{Adipocyte differentiation and chemical treatment}

To produce mature adipocytes, 3T3-L1 preadipocytes (American Type Culture Collection, Manassas, VA) were seeded on 12-well plates with growth medium to let the cells reach $100 \%$ confluence. Two days later, the cells were induced to become mature adipocytes with a cocktail adipogenic inducers, defined as day 0 (D0) for 2 days. Next, the cells were refreshed with the maintenance medium for an additional 2 days. The medium was finally replaced with growth medium for 10 more days. The cells were treated with relative chemicals from days 0 to 14 as follows: the non-induction group (N.I.); the AA2P alone treated group (N.I. + AA2P); the adipogenic group (I), $250 \mu \mathrm{M}$ AA2P group (I + AA2P); the $100 \mu \mathrm{M}$ EDHB group $(\mathrm{I}+\mathrm{EDHB})$; the $100 \mu \mathrm{M}$ EDHB plus $250 \mu \mathrm{M}$ AA2P group (I + EDHB + AA2P); the RNAi groups; the $100 \mu \mathrm{M}$ U0126 group (I + U0126); and the $100 \mu \mathrm{M}$ U0126 plus $250 \mu \mathrm{M}$ AA2P group (I + U0126 + AA2P). The experimental groups were shown in Fig. 1.

\section{Construction of lentiviral vector for silencing of mouse collagen VI a1 subunit expression}

Small hairpin RNA (shRNA) of the mouse collagen VI $\alpha 1$ subunit lentivirus gene transfer vector encoding the GFP sequence was designed. Different siRNAs were screened by co-transfection with mouse collagen VI $\alpha 1$ subunit cDNA plasmid into HEK293T cells with Lipofectamine 2000 (Invitrogen Corporation, Carlsbad, CA, USA). The optimal sequence of the siRNA against the mouse collagen VI $\alpha 1$ subunit (5'-ACCTATTCCGTGT ACCAAA-3') (GenBank gi: NM_009933), was then cloned into the plasmid GV 112, which encodes an HIVderived lentiviral vector containing a multiple cloning 


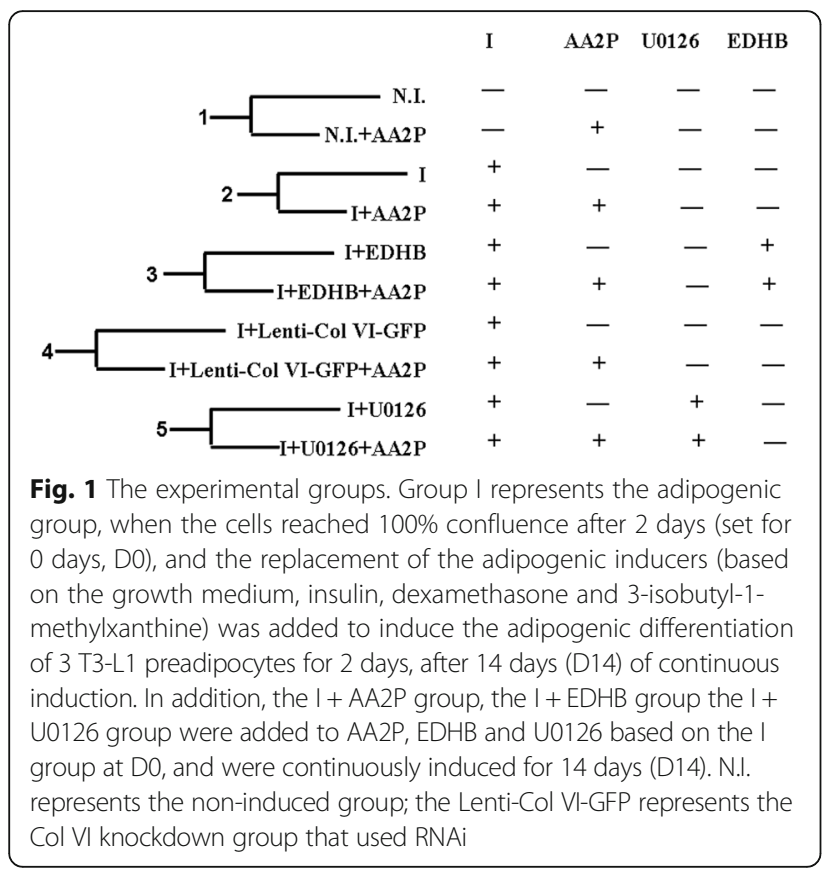

site for insertion of the shRNA constructs to be driven by an upstream U6 promoter and a downstream cytomegalovirus promoter with GFP. The resulting lentiviral vector containing mouse collagen VI $\alpha 1$ subunit shRNA was named Lenti-ColVI-GFP, and its sequence was confirmed by PCR and sequencing analysis. A negative control (NC) lentiviral vector (Lenti-GFP) containing NC shRNA was constructed by a similar process (NC lentivirus, 5'-TTCTCCGAACGTGTCACGT-3').

\section{Lentivirus siRNA gene transfection}

3T3-L1 preadipocytes were plated in 12-well plates overnight and infected with Lenti-ColVI-GFP by addition of lentivirus diluted into $0.5 \mathrm{ml}$ of Dulbecco's modified eagle medium (DMEM) containing 5\% NBCS and polybrene at an MOI of approximately 100 for $12 \mathrm{~h}$ at $37^{\circ} \mathrm{C}$. The medium was then replaced with growth medium until it reached $100 \%$ confluence. Adipogenic induction was initiated after $48 \mathrm{~h}$ of confluence. The controls were infected with NC lentivirus (I + Lenti-GFP).

\section{Oil red $\mathbf{O}$ staining}

The cells were stained with Oil Red O (Sigma-Aldrich) at the D3, D7 and D14 after induction for determining the degree of differentiation (the ratio of adipose cells). The lipid staining was quantified by extracting the dye with $100 \%$ isopropanol and measuring the absorbance at $520 \mathrm{~nm}$.

\section{RNA isolation and real-time quantitative reverse transcription (RT)-PCR}

Total RNA was extracted from 3T3-L1 cells at the D0, D3, D7 and D14 after induction using AxyPrep Total RNA Preparation Kit (Axygen, American) according to the manufacturer's protocol. RNA concentration was determined by optical density at $260 \mathrm{~nm}$ (OD 260) using Nanodrop (Thermo Scientific, MA, USA). Total RNA $(0.3 \mu \mathrm{g})$ was reverse-transcribed using First Strand cDNA Synthesis Kit (TOYOBO, FSK-100). The expression level of genes was assessed by quantitative RT-PCR. cDNA product $(0.2 \mu \mathrm{g})$ was amplified by quantitative PCR with SuperReal RT-PCR PreMix (SYBR Green) kit (Tiangen, China, FP204). The results are expressed as the fold increase relative to the controls after normalizing to the $\beta$-actin gene expression levels. The sequences of the gene-specific primers used are shown in Table 1.

\section{Western blotting}

Total cell proteins were isolated using a lysis buffer and sonicated as previously reported [30]. An aliquot containing $30 \mu \mathrm{g}$ of proteins was separated by electrophoresis on a $12 \%$ sodium dodecyl sulfate-polyacrylamide gel electrophoresis (SDS-PAGE) and was electrophoretically transferred to PVDF membranes (Millipore, Massachusetts, USA). The membranes were blocked in $10 \mathrm{mM}$ of Tris

Table 1 The sequences of the gene-specific primers

\begin{tabular}{|c|c|c|c|}
\hline Gene & GenBank No. & Primer & Length \\
\hline Collagen VI a1 & NM_009933 & $\begin{array}{l}\text { F: 5'-TGCCCTGTGGATCTATTCTTCG-3' } \\
\text { R: 5'-CTGTCTCTCAGGTTGTCAATG-3' }\end{array}$ & $107 \mathrm{bp}$ \\
\hline SREBP-1C & NM_011480 & $\begin{array}{l}\text { F: 5'-GTGAGCCTGACAAGCAATCA-3' } \\
\text { R: 5'-GGTGCCTACAGAGCAAGAG-3' }\end{array}$ & $102 \mathrm{bp}$ \\
\hline C/EBP $\beta$ & NM_009883 & $\begin{array}{l}\text { F: 5'-ACACGGGACTGACGCAACAC-3' } \\
\text { R: 5'-AACCCCGCAGGAACATCTIT-3' }\end{array}$ & 78 bp \\
\hline PPARY2 & NM_011146 & $\begin{array}{l}\text { F: 5'-GTCATCCTGCTCTTCTTTCTCG-3' } \\
\text { R: 5'-ATGGCGTCCCTTCTCCTGT-3' }\end{array}$ & $115 \mathrm{bp}$ \\
\hline C/EBPa & NM_007678 & $\begin{array}{l}\text { F: 5'-CAGGAGGAAGATACAGGAAGC-3' } \\
\text { R: 5'-TCTCCATGAACTCACCCAGG-3' }\end{array}$ & $229 \mathrm{bp}$ \\
\hline$\beta$-actin & NM_007393 & $\begin{array}{l}\text { F: 5'-CCTCTATGCCAACACAGTGC-3' } \\
\text { R: 5'-GTACTCCTGCTTGCTGATCC-3' }\end{array}$ & $193 \mathrm{bp}$ \\
\hline
\end{tabular}


buffer solution (TBS, pH 7.5) containing $0.1 \%$ Tween 20 (TBS-T) and 5\% BSA (Beyotime, Shanghai, China) for $1 \mathrm{~h}$ at room temperature. After washing with TBS-T, the membranes were incubated with primary antibodies (anticollagen VI $\alpha 1$ (Abcam, Cambridge, MA), anti-ERK1/2, anti-phospho-ERK1/2, anti-PPAR $\gamma$, anti-C/EBP $\alpha$ (all from Cell Signalling, Danvers, MA), anti-SREBP-1c and anti-C/ EBP $\beta$ (all from Santa Cruz, California, USA) overnight at $4{ }^{\circ} \mathrm{C}$. After extensive washing, the membranes were further incubated with the corresponding secondary antibodies (horseradish peroxidase-conjugated goat anti-mouse and goat anti-rabbit IgG, Jackson, PA, USA) for $1.5 \mathrm{~h}$ at a dilution of 1:10,000. The proteins were detected by the Immobilon Western Chemiluminescent HRP Substrate (Millipore, MA, USA) and were displayed on X-ray film (Fujifilm, Tokyo, Japan). QuantiScan 3.0 was used to analyze the gray value of the protein blot. The gray value of the target protein blot divided by the gray value of the internal reference GAPDH and/or $\alpha$-tubulin (GenScript, Piscataway, NJ, USA) represents the relative expression of the target protein.

\section{Statistical analysis}

Data were analyzed using SPSS version 19.0 software (SPSS Inc., Chicago, IL, USA) and two tailed in tests. Each experiment was performed in at least triplicate. The data are expressed as the mean \pm SEM with GraphPad Prism 5. Differences between groups were analyzed by conducting one-way analysis of variance (ANOVA) followed by Student's $\mathrm{t}$ test. ${ }^{*} P<0.05$ was considered statistically significant and significant between groups.

\section{Results}

\section{AA2P promotes adipogenesis of 3T3-L1 cells}

Consistent with a previous study [18], AA2P alone could induce the adipogenesis of 3T3-L1 mouse preadipocytes without adipogenic induction, although this effect was weaker. On the other hand, lipid droplets began to be accumulate from D3 when the cells were treated with adipogenic inducer. With the progress of adipogenesis, lipid droplets gradually increased and reached a peak at D7. Compared with this group, more evident adipogenesis was found in the I + AA2P group (Fig. 2a).

Further studies had shown that AA2P significantly upregulated the expression of collagen VI, SREBP-1c, C/ EBP $\beta$, PPAR 22 and $C / E B P \alpha(P<0.05)$ in both the noninduction group and the adipogenic group. However, their expression in the late stage (D8-D14) tended to be consistent, except for PPAR 2 in the adipogenic group (Fig. 2bd). The total ERK1/2 remained essentially unchanged; however, the ERK1/2 phosphorylation $(P<0.05)$ was gradually reduced, and there was almost no expression in the late stage of adipogenesis. In the early and middle stages of adipogenesis, ERK1/2 phosphorylation $(P<0.05)$ in the AA2P treatment group was significantly lower than that in the adipogenic group. These data suggest that AA2P may attenuate the ERK1/2 phosphorylation to up-regulate the expression of collagen VI and further up-regulate the expression of SREBP-1c, C/EBP $\beta$, PPAR $\gamma 2$ and $\mathrm{C} / \mathrm{EBP} \alpha$ during the adipogenesis of 3T3L1 cells. The further question is raised: is the above mechanism by which AA2P promotes the adipogenesis of 3T3-L1 cells correct?

\section{Inhibition of collagens affects the adipogenesis of 3T3-L1 cells}

To confirm the effect of collagens on adipogenesis, $100 \mu \mathrm{M}$ of EDHB was added on the overall stage of adipogenesis or after treatment with AA2P. The results showed that EDHB completely inhibited the adipogenesis of 3T3-L1 cells (Fig. 3a, I+EDHB group), indicating that collagens played an important role in the process. Notably, the inhibitory effect of EDHB on cell adipogenesis was partially reversed by AA2P (Fig. 3a, I+EDHB + AA2P group).

It has been reported that the failure of the hydroxylation of collagen subunits will result in a non-functional degradable protein [11]. We examined the expression of SREBP-1c, C/EBP $\beta$, PPAR $\gamma 2$ and C/EBP $\alpha$. The results showed that EDHB significantly reduced the assembly and secretion of collagen VI and inhibited the expression of SREBP-1c, C/EBP $\beta$, PPAR 22 and C/EBP $\alpha(P<0.01)$ (Fig. 3b-d). In contrast, the expression of collagen VI was significantly up-regulated at the transcriptional level (Fig. 3b, I+EDHB group). The possible reason for this is that EDHB inhibited the assembly of collagen subunits without inhibiting the transcription level, and collagen VI was up-regulated by positive feedback after the synthesis of the collagen VI was inhibited.

Moreover, our results showed that EDHB could significantly increase the ERK1/2 phosphorylation $(P<0.01)$, and the expression trend was opposite with collagen VI (Fig. 3c and d). This finding suggested that there may be interactions between the two molecules. Additionally, ERK1/2 phosphorylation $(P<0.01)$ was significantly decreased after treating with AA2P, indicating that AA2P could attenuate ERK phosphorylation. These data demonstrate that AA2P may attenuate ERK phosphorylation to up-regulate the expression of collagen VI during the adipogenesis of 3T3-L1 cells. The data shows that adipogenesis was inhibited when the expression of type I to VI collagen was suppressed, and it was reversed partially when treated with AA2P, indicating that collagens play a key role during adipogenesis. Another question is raised: is collagen VI among the types of collagen that have more importance? 


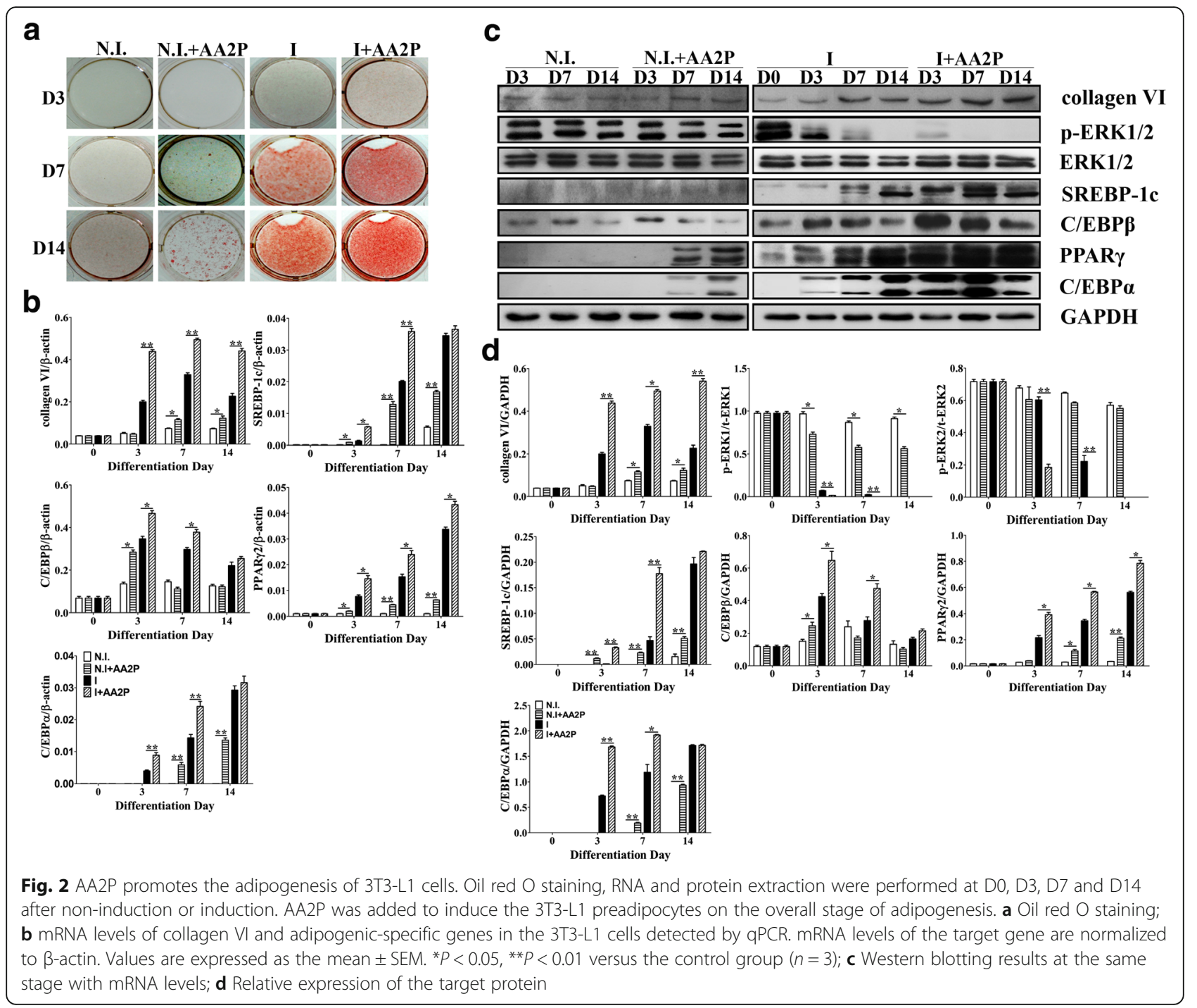

\section{Knockdown of collagen VI inhibits the adipogenesis of 3T3-L1 preadipocytes}

To further verify the role of collagen VI during adipogenesis, collagen VI knockdown was performed by using RNAi. The results showed that knockdown of collagen VI significantly inhibited the adipogenesis of 3T3-L1 cells (Fig. 4a and b) due to inhibited the expression of SREBP-1c, C/EBP $\beta$, PPAR 2 and C/EBP $\alpha(P<0.01)$ (Fig. 4c-e). The knockdown efficiency of collagen VI was approximately $65.3 \%$ (see Additional file 1), and the ratio of adipose cells was reduced by approximately $51.6 \%$. The ratio was partially restored after treatment with AA2P, indicating that AA2P promoted the synthesis of collagen VI, which corroborated previous reports.

We further detected the phosphorylation of ERK1/2. The results showed that knockdown of collagen VI can significantly promote the phosphorylation of ERK1/2, especially ERK1 phosphorylation $(P<0.05)$; therefore, the expression of PPAR 2 in the Lenti-ColVI-GFP group was significantly lower $(P<0.01)$ than that in the control group. The ERK1 phosphorylation was significantly down-regulated $(P<0.05)$ after treatment with AA2P at D3, further demonstrating that AA2P could attenuate ERK1/2 phosphorylation, especially the ERK1 phosphorylation (Fig. 4d and e). These data further demonstrate the interaction between collagen VI and ERK signaling and that AA2P attenuates the ERK1/2 phosphorylation to up-regulate the expression of collagen VI during the adipogenesis of 3T3-L1 cells.

\section{Inhibiting the ERK $1 / 2$ phosphorylation affects the adipogenesis of 3T3-L1 cells}

To further verify the effect of ERK signaling on adipogenesis, a specific inhibitor of MEK1 and MEK2, U0126, which also activated ERK (ERK1/2 phosphorylation), was added at the overall stage of adipogenesis or after 

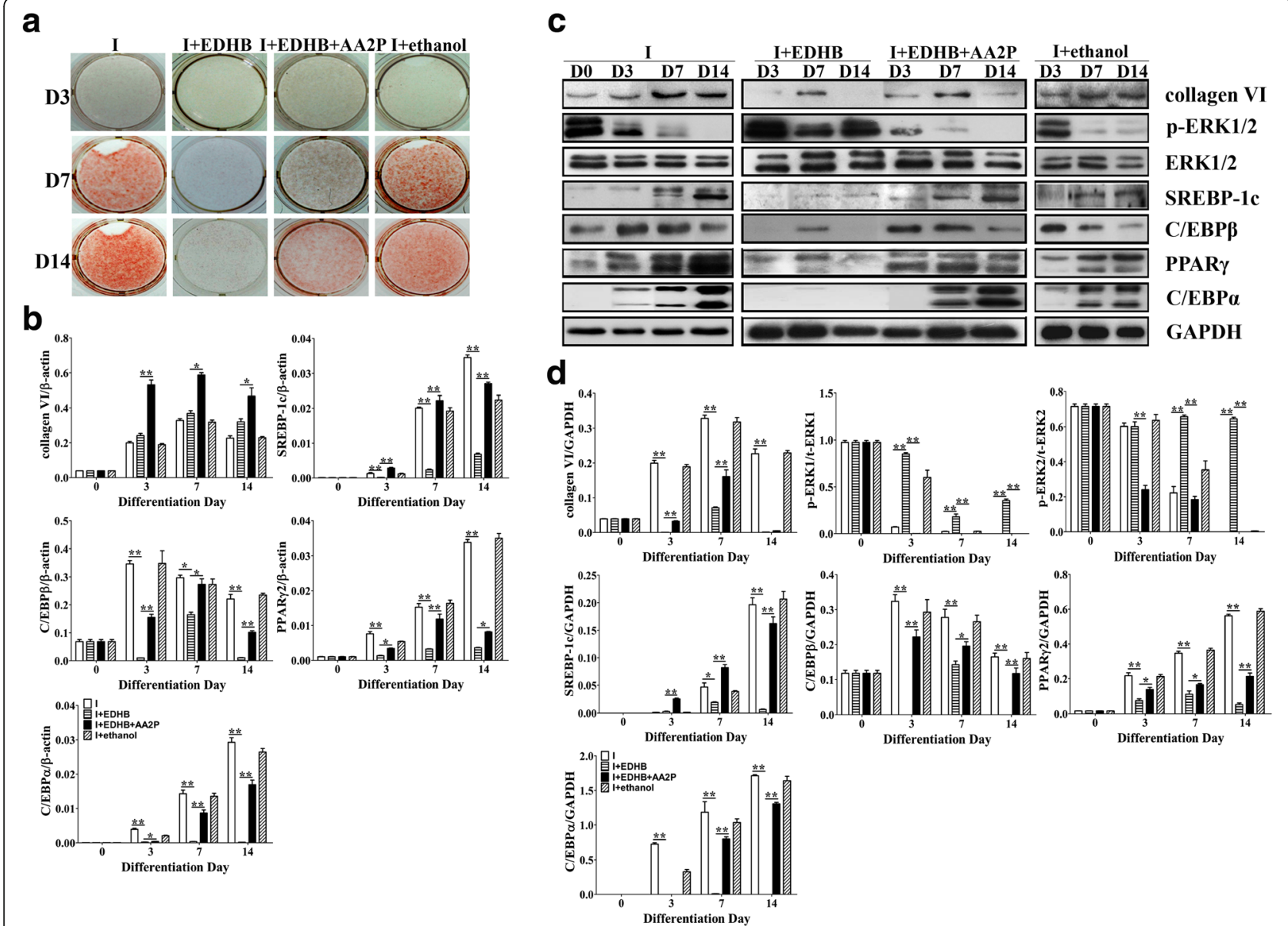

Fig. 3 Inhibition of collagen affects the adipogenesis of the 3T3-L1 cells. Oil red $O$ staining, RNA and protein extractions were performed at D0, D3, D7 and D14 after induction. EDHB was added at the overall stage of adipogenesis or after treating with AA2P. a Oil red O staining; $\mathbf{b}$ mRNA levels of collagen $\mathrm{VI}$ and adipogenic-specific genes in the 3T3-L1 cells detected by qPCR. mRNA levels of the target gene are normalized to $\beta$ actin. Values are expressed as the mean \pm SEM. ${ }^{*} P<0.05$, ${ }^{* *} P<0.01$ versus the control group $(n=3)$; $\mathbf{c}$ Western blotting results at the same stage with mRNA levels; d Relative expression of the target protein

treatment with AA2P. U0126 completely inhibited the adipogenesis of 3T3-L1 cells (Fig. 5a) and inhibited the expression of $\mathrm{C} / \mathrm{EBP} \beta$, PPAR $\gamma$ and $\mathrm{C} / \mathrm{EBP} \alpha(P<0.01)$ (Fig. 5c-e). Furthermore, we added U0126 at D3 and D7. The results showed that ERK1/2 phosphorylation mainly regulated the adipogenesis of 3T3-L1 cells in the early and middle stage of adipogenesis (Fig. 5b).

The above results appear to be contrary to our expected results. However, previous studies have shown that ERK1/2 phosphorylation in the early stage of adipogenesis is essential for C/EBPs and PPAR $\gamma$ transcription, while PPAR $\gamma$ is a downstream ERK1/2 molecule and the activation of ERK1/2 could phosphorylate PPAR $\gamma$, leading to a reduction in its transcriptional activity, thereby inhibiting adipogenesis [31]. Moreover, our data suggest that the lack of ERK signaling could not transmit the collagen signal into the cells.
Collagen VI significantly increased $(P<0.05)$ after U0126 treatment, further indicating that there is an interaction between collagen VI and ERK signaling. Moreover, AA2P could regulate the expression of collagen through ERK signaling (Fig. 5c-e). Surprisingly, the expression of SREBP-1c was not inhibited by U0126 in the early and middle stage of adipogenesis. However, it was inhibited in the late stage of adipogenesis. This may be suggested the fact that the ERK1/2 phosphorylation inhibits the expression of SREBP-1c. These data further demonstrate that AA2P could attenuate ERK1/2 phosphorylation to up-regulate the expression of collagen VI, and that ERK signaling is necessary to transmit the collagen signal into the cells.

\section{Discussion}

It is known that VC is not only an important antioxidant also an indispensable nutrient. Recently, with the 


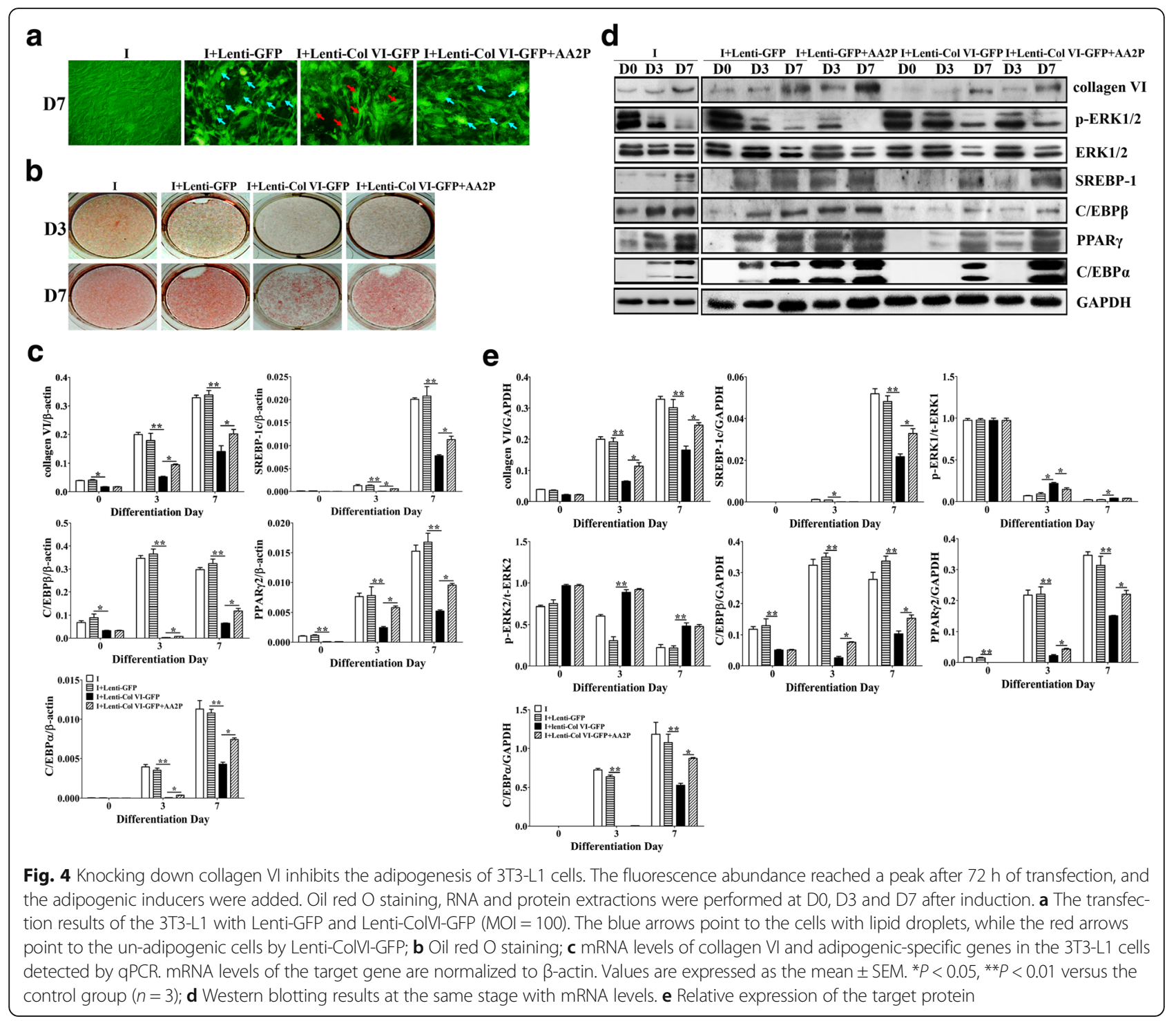

development of induced pluripotent stem cell technology, $\mathrm{VC}$ has been used to improve the efficiency of induced pluripotent stem cells [32]. Studies have shown that VC can regulate the adipogenesis of preadipocytes, but its regulatory mechanisms need to be further studied. Therefore, we used AA2P, which is a stable derivative of VC that possesses similar biological properties, to explore the mechanism of AA2P to regulate the adipogenesis of 3T3$\mathrm{L} 1$ preadipocytes in vitro.

It has been reported that $200 \mu \mathrm{M}$ and $250 \mu \mathrm{M}$ of AA2P could stimulate the lipid accumulation of 3T3-L1 cells and promote the adipogenesis of BMSCs [18, 21]. Consistently, our results showed that AA2P could significantly promote the adipogenesis of 3T3-L1 preadipocytes in a dose-dependent manner (see Additional file 2), and that the concentration of $250 \mu \mathrm{M}$ is more potent without toxicity to the cells.
We next detected the expression of adipocyte specific factors, such as SREBP-1c, C/EBP $\beta, C / E B P \alpha$ and PPAR $\gamma$. Consistent with a previous study [19], our data showed that AA2P could significantly up-regulate the expression of these factors. However, the expression of SREBP-1c, $\mathrm{C} / \mathrm{EBP} \beta$ and $\mathrm{C} / \mathrm{EBP} \alpha$ in the AA2P treatment group was consistent with that in the untreated adipogenic group at the late stage of adipogenesis, indicating that AA2P could accelerate the expression of SREBP-1c, C/EBP $\beta$ and $\mathrm{C} / \mathrm{EBP} \alpha$ and maintain the expression of PPARy. Therefore, the results raise the question of how AA2P accelerates or up-regulates the expression of these factors.

It has been reported that the remodeling of ECM has increased flexibility and changes the connection among collagens, promoting the transcription of adipocyte specific genes, such as C/EBP $\beta$ and PPAR $\gamma$ [5]. To determine the 


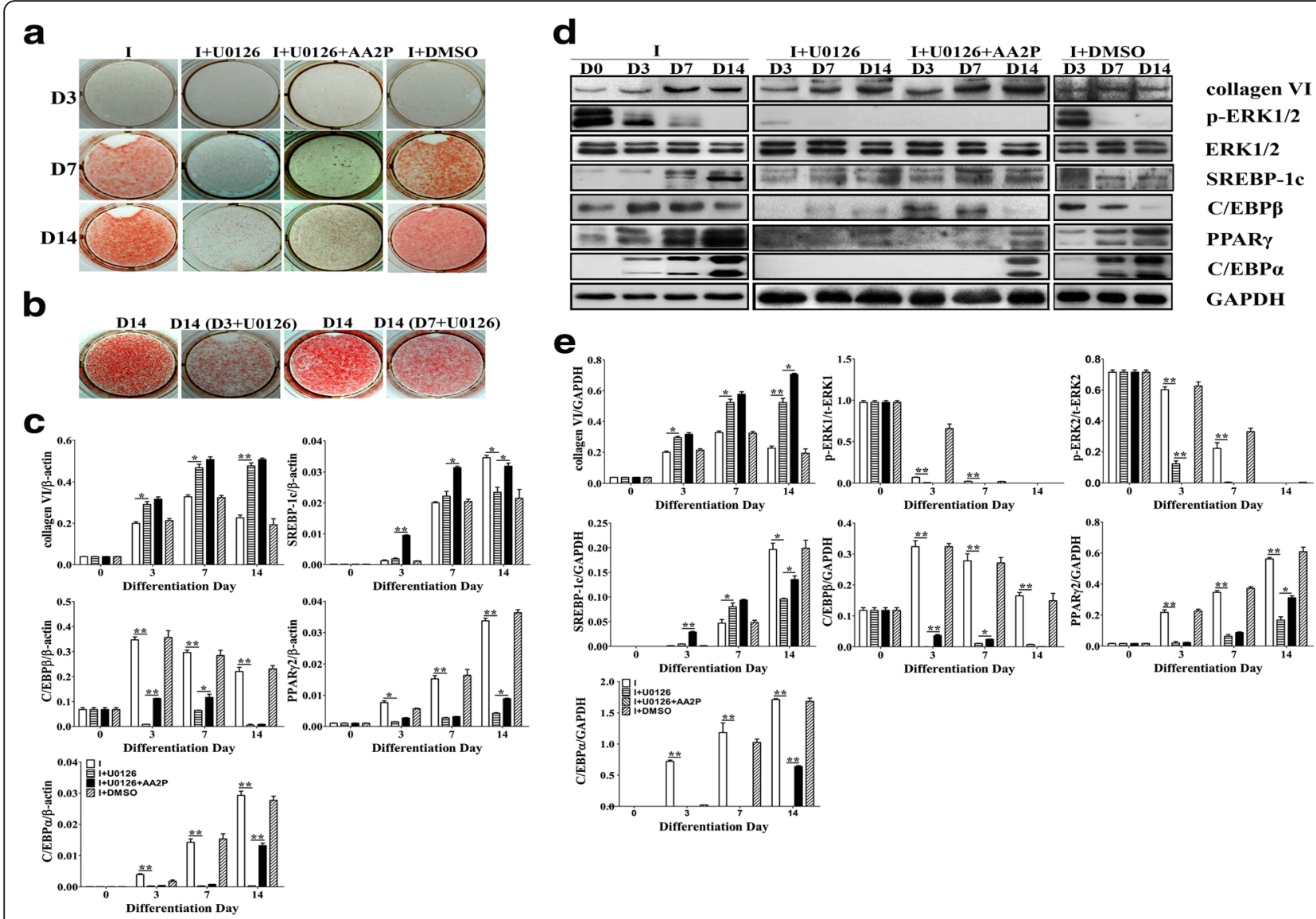

Fig. 5 Inhibiting ERK1/2 phosphorylation affects the adipogenesis of 3T3-L1 cells. Oil red O staining, RNA and protein extraction were performed at D0, D3, D7 and D14 after induction. U0126 (100 $\mu \mathrm{M})$, a specific inhibitor of ERK1/2 phosphorylation, was added at the overall phase, after treatment with $250 \mu \mathrm{M}$ of AA2P (from D0 to D14), or at the early (form D3 to D14) or middle (form D7 to D14) stage of adipogenesis. a Oil red O staining; $\mathbf{b}$ The effect of U0126 on adipogenesis was added at the early (D3) and middle stage (D7), Oil red O staining was performed at D14 after induction; c mRNA levels of collagen VI and adipogenic-specific genes in the 3T3-L1 cells detected by qPCR. mRNA levels of the target gene are normalized to $\beta$-actin. Values are expressed as the mean \pm SEM. ${ }^{*} P<0.05,{ }^{*} P<0.01$ versus the control group $(n=3)$; $\mathbf{d}$ Western blotting results at the same stage with mRNA levels; e Relative expression of the target protein

roles played by collagens in adipogenesis, we first added EDHB, which is an ideal inhibitor of collagens synthesis. Our data showed that EDHB significantly inhibited the adipogenesis of 3T3-L1 cells by down-regulating the expression of SREBP-1c, C/EBPs and PPAR $\gamma$.

Collagen VI plays a vital role in the connection among collagens [3]. It was reported that the TG content of BIP is reduced to $50 \%$ by EDHB at a concentration of $0.1 \mathrm{mM}$. Moreover, the ratio of adipose cells was restored to $31.5 \%$ when cells that were treated with EDHB were cultured on dishes coated with collagen VI [1], may indicating that collagen VI plays a primary role in adipogenesis. Based on this observation, we focused on the expression of collagen VI. Our data showed that AA2P could significantly upregulate the expression of collagen VI. Moreover, the mRNA of collagen VI was up-regulated in contrast with the protein of collagen VI after treatment with EDHB.
The reason for this is that EDHB only inhibits the assembly and secretion of collagens and does not inhibit the expression of collagen genes, indicating the role of EDHB on collagen synthesis. EDHB significantly inhibited the adipogenesis of 3T3-L1 cells by down-regulating the assembly and secretion of collagen VI to down-regulate the expression of adipocyte specific factors. After treating with AA2P, the expression of collagen VI was up-regulated accompanied by the up-regulation of adipocyte-specific factors, which led to the recovery of the ratio of adipose cells by approximately $50.4 \%$. The reason for this was that EDHB did not completely inhibit the synthesis of collagen VI. AA2P partially promoted the synthesis of collagen and up-regulated the expression of the adipocyte specific factors, resulting in the partially restored adipogenesis of 3T3-L1 cells. Our Western blot results validated the above speculation. 
In addition, we only detected collagen VI, not detect other collagens (type I-V), so that we did not know how much remained after adding EDHB (I + EDHB). We specifically knocked down collagen VI, and the ratio of adipose cells was significantly higher than that of the I+ EDHB group. Furthermore, the efficiency of AA2P rescuing the ratio of adipose cells in the I + Lenti-ColVIGFP + AA2P group was significantly higher than that in the I + EDHB + AA2P group. Taken together, we found that other collagens (type I-V) also play a role in adipogenic differentiation, while collagen VI plays a major role in adipogenic differentiation of 3T3-L1 preadipocytes.

Nakajima [1, 2] showed that when confluent bovine intramuscular preadipocytes (BIP) were stimulated with adipogenic medium, there was a significant accretion on the cell surface of the type I-VI collagens, especially type $\mathrm{V}$ and VI. When exogenous collagens were supplied to make up for the lack of endogenous products, cultured EDHB-treated cells on type V and VI collagen-coated dishes were the only ones among six collagens to accumulate more TG, with recovery rates of $23.2 \%$ and $31.5 \%$, respectively. There was no significant positive or negative effect on TG accumulation in other types of collagens [1]. The above results confirm our previous finding that other collagens (type I-V) also play a role in adipogenic differentiation, while collagen VI plays a major role in adipogenic differentiation.

To further verify the regulation of collagen VI on the adipogenesis of 3T3-L1 cells, we used RNAi to knockdown the $\alpha 1$ subunit of collagen VI, which significantly inhibited adipogenesis of 3T3-L1 cells by downregulating the expression of SREBP-1c, C/EBP $\beta, C / E B P \alpha$ and PPAR $\gamma$. However, after treating with AA2P, the adipocyte-specific factors and adipogenesis were restored partially. Moreover, the ratio of adipose cells in the Lenti-ColVI-GFP group was higher than that in the $\mathrm{EDHB}+\mathrm{AA} 2 \mathrm{P}$ group, indicating that AA2P promoted the expression of adipocyte specific factors by upregulating the synthesis of the collagen VI.

It has been reported that VC could up-regulate the expression of collagen I and III by inhibiting the phosphorylation of ERK1/2 [29, 33-37]. Consistent with previous studies $[28,29]$, our results indicated that AA2P did not affect the expression of ERK1/2, while it significantly down regulated the ERK1/2 phosphorylation in the early and middle stage of adipogenesis (D0-D7). Moreover, the results showed that the earlier the addition of U0126, the greater the inhibition of adipogenesis (Fig. $5 \mathrm{~b})$, further indicating that the ERK signaling plays an important role in the early stage of adipogenesis.

After treating the 3T3-L1 cells with EDHB, the expression trend of the collagen VI was opposite that of the ERK1/2 phosphorylation. In other words, the ERK1/2 phosphorylation was inhibited by the expression of collagen VI. Further studies showed that the ERK1/2 phosphorylation was significantly up-regulated after knockdown of collagen VI. ERK1/2 phosphorylation was decreased after treating with AA2P, while it was significantly higher than that of the Lenti-GFP control group. Bost et al. [38] reported that ERK1 plays a major role in adipogenesis. Consistently, our results showed that knocking down collagen VI mainly impacted ERK1 phosphorylation.

To further verify the role of ERK signaling, we treated the cells with U0126, an inhibitor of ERK1/2 phosphorylation, during 3T3-L1 cells adipogenesis. Previous studies have shown that the ERK1/2 phosphorylation in the early stage of adipogenesis is essential for C/EBPs and PPAR $\gamma$ transcription, but PPAR $\gamma$ is a downstream molecule of ERK1/2 and ERK1/2 phosphorylation could phosphorylate PPAR $\gamma$. As result reduce in its transcriptional activity, thereby inhibiting adipogenesis [31, 39]. Therefore, the ERK1/2 phosphorylation decreased gradually with adipogenesis.

In this study, we have shown the significantly upregulated expression of collagen VI after completely inhibiting ERK1/2 phosphorylation by U0126. On the contrary, the adipocyte specific gene factors were significantly down-regulated. This seems to be contrary to our hypothesis. However, AA2P only attenuates ERK1/2 phosphorylation, rather than completely inhibiting it, such as in U0126. In addition, it was surprising that the expression of SREBP-1c was not inhibited by U0126 in the early and middle stage of adipogenesis. However, it was inhibited in the late stage of adipogenesis. This issue needs to be further studied.

Inhibition of ERK1/2 with U0126 results in the inhibition of adipogenesis, which is rescued by AA2P. This is puzzling since the $\mathrm{p}$-ERK $1 / 2$ levels tended to decrease with increased adipogenesis.

Previous studies have shown that ERK1/2 phosphorylation in the mitotic clonal expansion stage of adipogenesis is essential for $\mathrm{C} / \mathrm{EBP} \beta$, while PPAR $\gamma$ is a downstream molecule of ERK1/2, and the activation of ERK $1 / 2$ could phosphorylate PPAR $\gamma$; therefore, the p-ERK1/2 levels tended to decrease with increased adipogenesis [31, 39]. The present data showed that ERK1/2 phosphorylation mainly regulated the adipogenesis of 3T3-L1 cells in the early and middle stage of adipogenesis, especially in the early stage of adipogenesis (Fig. 5b).

Our results showed that AA2P promotes the adipogenesis of the 3T3-L1 cells by attenuating ERK signaling to up-regulate collagen VI. In the absence of p-ERK $1 / 2$, AA2P could still partially rescue the inhibitory effect of U1026 on the adipogenic differentiation of 3T3-L1 preadipocytes. The possible reason for this is that AA2P could promote the adipogenic differentiation of preadipocytes by other pathways. 


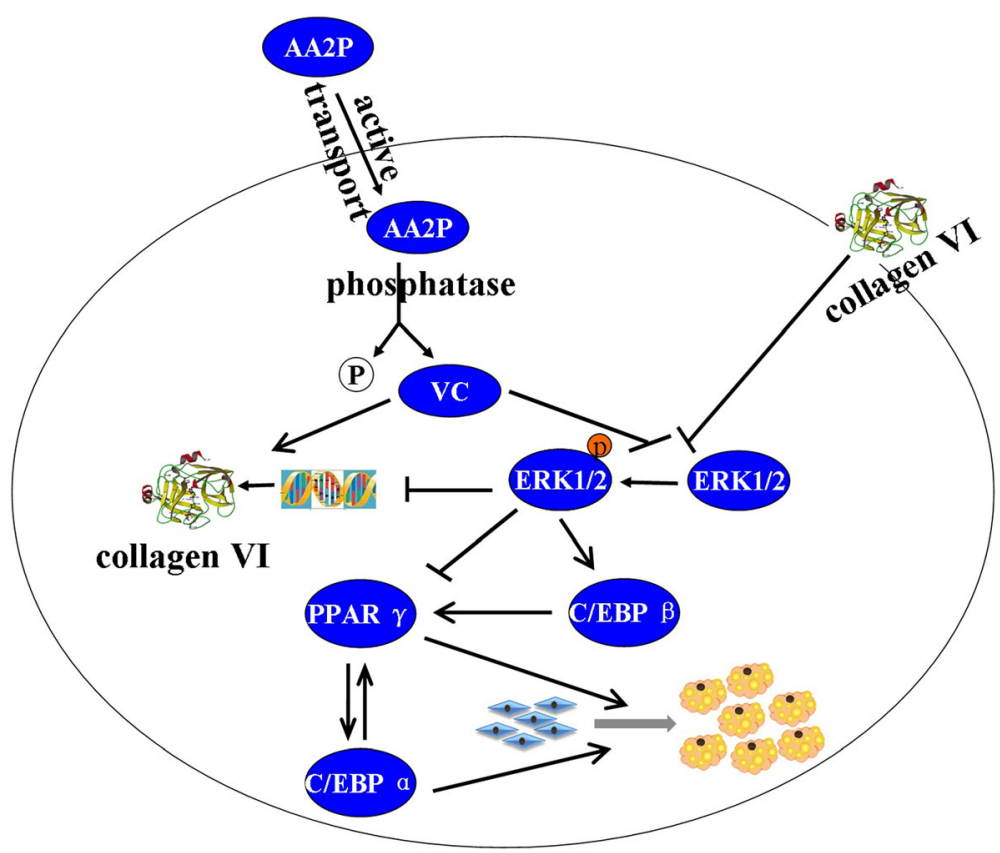

Fig. 6 The possible mechanism of AA2P promoting the adipogenesis of 3T3-L1 cells. AA2P enters the cells by active transport and plays two roles. First, AA2P promotes the assembly and secretion of collagen $\mathrm{VI}$ as the hydroxyl factor co-enzyme, and then down-regulates the phosphorylation of ERK1/2. Second, VC down regulates ERK1/2 phosphorylation directly by a certain mechanism

$\mathrm{VC}$ has an important epigenetic regulatory function. Yin et al. [40] have shown that VC could enhance the ten-eleven translocation (Tet)-mediated oxidation of 5methylcytosine to 5-hydroxymethylcytosine and promote the demethylation of DNA, thereby activating gene expression. Further studies [41] have shown that PPARy could guide the local demethylation of its binding site (PPAR response elements, PPREs) and activate the genes involved in adipogenesis (such as perilipin), promoting the adipogenic differentiation of 3T3-L1 preadipocytes. It could be speculated that AA2P also promotes the adipogenic differentiation of 3T3-L1 preadipocytes by promoting the expression of PPAR $\gamma$ and acting as a cofactor of Tet to promote the expression of the genes involved in adipogenesis. However, the role of this pathway in promoting the adipogenic differentiation of 3T3-L1 preadipocytes is relatively weak. Therefore, AA2P promotes the adipogenesis of 3T3-L1 cells by mainly attenuating ERK signaling to up-regulate collagen VI.

\section{Conclusions}

Our data indicated that AA2P promotes 3T3-L1 preadipocyte adipogenic differentiation. The speculative mechanism of effects of AA2P on cell adipogenesis is as follows: $\mathrm{VC}$ promoted the synthesis and secretion of collagen VI as the hydroxyl factor co-enzyme and down-regulated the ERK1/2 phosphorylation to promote the adipogenesis of the preadipocytes by up-regulating the expression of adipocyte-specific factors, such as SREBP-1c, C/EBP $\beta$, PPAR $\gamma$ and C/EBP $\alpha$; AA2P up-regulated the expression of collagen VI by attenuating ERK1/2 phosphorylation, further up-regulating adipocyte-specific factors, and finally promoting the adipogenesis of 3T3-L1 cells (Fig. 6). It is noteworthy that VC accelerated the lipid accumulation on 3T3-L1 cells, at least in part by attenuating the ERK1/2 phosphorylation to up-regulate collagen VI. Further work need to be down carefully whether VC plays similar role in vivo, since it was reported that $\mathrm{VC}$ has dual function in adipogenesis in vivo.

\section{Additional files}

Additional file 1: Screened the $\mathrm{MOI}$ value of the lentivirus and detected the knockdown efficiency of Col VI: a $5 \times 104$ 3T3-L1 preadipocytes $/ \mathrm{ml}$ were plated in 12-well plates overnight and infected with Lenti-ColVI-GFP lentivirus by addition of lentivirus diluted into $0.5 \mathrm{ml}$ of Dulbecco's modified eagle medium (DMEM) containing 5\% NBCS and polybrene at the $\mathrm{MOI}$ of 0 , 20, 50 and 100 for $12 \mathrm{~h}$ at $37^{\circ} \mathrm{C}$. The medium was then replaced with growth medium until it reached $100 \%$ confluence. Adipogenic induction was initiated after $48 \mathrm{~h}$ of confluence. The controls were infected with NC lentivirus (I + Lenti-GFP); b After screening out the optimal MOI value of the lentivirus, we detected the knockdown efficiency of Col VI using real-time quantitative reverse transcription. Values are expressed as the mean $\pm \mathrm{SEM}$. ** $P<0.01$ versus the control group $(n=3)$. (PDF $234 \mathrm{~kb}$ )

Additional file 2: The effect of AA2P on adipogenesis is dosedependent: a 3T3-L1 preadipocytes were seeded on 12-well plate with growth medium (DMEM, 10\% new-born calf serum, penicillin $(100 \mathrm{U} / \mathrm{ml})$, streptomycin (100 mg/mL) and $2 \mathrm{mM} \mathrm{L-glutamine)} \mathrm{to} \mathrm{let} \mathrm{the} \mathrm{cells}$ reached $100 \%$ confluent. Two days later, the cells were induced for 
mature adipocytes with cocktail adipogenic induction medium (growth medium supplemented with $1.7 \mu \mathrm{M}$ insulin) for an additional 2 days. The medium was finally replaced with growth medium for 10 more days. AA2P was added from the D0 to D14 at final concentrations of 0,100 , 250 and $500 \mu \mathrm{M}$, respectively; $\mathrm{b}$ On day 14 , the cells were stained with Oil red O; The lipid the staining was quantified by extracting the dye with $100 \%$ isopropanol and measuring the absorbance at $520 \mathrm{~nm}$. Values are expressed as the mean \pm SEM. ${ }^{*} P<0.05$ versus the control group $(n=3)$ (PDF $1599 \mathrm{~kb})$

\section{Abbreviations}

AA2P: L-ascorbic acid-2-phosphate; BIP: Bovine intramuscular preadipocytes cell line; ColVI: Collagen VI; DMSO: Dimethyl sulfoxidein; ECM: Extracellular matrix; EDHB: Ethyl-3, 4-dihydroxybenzoate; ERK: Extracellular signal-regulated kinase; GFP: Green fluorescent protein; I: Induction; MAPK: Mitogen-activated protein kinase; N.I.: Non-induction; TG: Triglyceride; VC: Vitamin C

\section{Acknowledgements}

This work was supported in part by grants from the Natural Science Foundation of China (81070685), Science and Technology Projects of Shandong Province (J06 L11) and Natural Science Foundation of Shandong Province to JP, and Major Key Science and Technology Project in Shandong Province (2015ZDJS04003) to GL.

\section{Availability of data and materisals}

Please contact author (JP) for data or material requests.

\section{Authors' contributions}

Author contributions: $\mathrm{CL}, \mathrm{KH}, \mathrm{GL}, \mathrm{PW}$, and JP designed experiments. $\mathrm{CL}, \mathrm{KH}$, $\mathrm{CL}, \mathrm{CG}, \mathrm{ZS}$ and JP collected data. CL, KH, GL, PW, CL, CG, ZS and JP analyzed data. $C L, K H, G L, P W$ and JP wrote the manuscript. All authors reviewed and edited the final manuscript. All authors read and approved the final manuscript.

\section{Ethics approval and consent to participate}

The authors consent to the publication of the data.

\section{Consent for publication}

The authors consent to the publication of the data.

\section{Competing interests}

No conflicts of interest, financial or otherwise, are declared by the authors.

\section{Publisher's Note}

Springer Nature remains neutral with regard to jurisdictional claims in published maps and institutional affiliations.

Received: 12 May 2017 Accepted: 7 December 2017

Published online: 28 December 2017

\section{References}

1. Nakajima I, Muroya S, Tanabe RI, Chikuni K. Positive effect of collagen V and $\mathrm{VI}$ on triglyceride accumulation during differentiation in cultures of bovine intramuscular adipocytes. Differentiation. 2002;70(2-3):84-91.

2. Nakajima I, Muroya S, Tanabe RI, Chikuni K. Extracellular matrix development during differentiation into adipocytes with a unique increase in type $V$ and VI collagen. Biol Cell. 2002;94(3):197-203.

3. Chun TH. Peri-adipocyte ECM remodeling in obesity and adipose tissue fibrosis. Adipocyte. 2012;1(2):89-95.

4. Ojima K, Oe M, Nakajima I, Muroya S, Nishimura T. Dynamics of protein secretion during adipocyte differentiation. FEBS Open Bio. 2016;6(8):816-26.

5. Selvarajan S, Lund LR, Takeuchi T, Craik CS, Werb ZA. Plasma kallikreindependent plasminogen cascade required for adipocyte differentiation. Nat Cell Biol. 2001;3(3):267-75.

6. Bonaldo P, Braghetta P, Zanetti M, Piccolo S, Volpin D, Bressan GM, Collagen VI. Deficiency induces early onset myopathy in the mouse: an animal mode for Bethlem myopathy. Hum Mol Genet. 1998;7(13):2135-40.

7. Irwin WA, Bergamin N, Sabatelli P, Reggiani C, Megighian A, Merlini L, et al. Mitochondrial dysfunction and apoptosis in myopathic mice with collagen VI deficiency. Nat Genet. 2003;35(4):367-71.
8. Chen P, Cescon M, Megighian A, Bonaldo P, Collagen VI. Regulates peripheral nerve myelination and function. FASEB J. 2014;28(3):1145-56.

9. lyengar P, Espina V, Williams TW, Lin Y, Berry D, Jelicks LA, et al. Adipocytederived collagen $\mathrm{VI}$ affects early mammary tumor progression in vivo, demonstrating a critical interaction in the tumor/stroma microenvironment. J Clin Invest. 2005;115(5):1163-76.

10. Khan T, Muise ES, lyengar P, Wang ZV, Chandalia M, Abate N, et al. Metabolic dysregulation and adipose tissue fibrosis: role of collagen VI. Mol Cell Biol. 2009;29(6):1575-91.

11. Kivirikko Kl, Myllylä R, Pihlajaniemi T. Protein hydroxylation: prolyl 4hydroxylase, an enzyme with four cosubstrates and a multifunctional subunit. FASEB J. 1989;3(5):1609-17.

12. Murad S, Grove D, Lindberg KA, Reynolds G, Sivarajah A, Pinnell SR. Regulation of collagen synthesis by ascorbic acid. Proc Natl Acad Sci U S A. 1981:78(5):2879-82.

13. Alcain FJ, Buron MI. Ascorbate on cell growth and differentiation. J Bioenerg Biomembr. 1994:26(4):393-8.

14. Takahashi T, Lord B, Schulze PC, Fryer RM, Sarang SS, Gullans SR, et al. Ascorbic acid enhances differentiation of embryonic stem cells into cardiac myocytes. Circulation. 2003;107(107):1912-6.

15. Cao N, Liu Z, Chen Z, Wang J, Chen T, Zhao X, et al. Ascorbic acid enhances the cardiac differentiation of induced pluripotent stem cells through promoting the proliferation of cardiac progenitor cells. Cell Res. 2011;22(1):219-36.

16. Sato H, Takahashi M, Ise H, Yamada A, Hirose S, Tagawa Y, et al. Collagen synthesis is required for ascorbic acid-enhanced differentiation of mouse embryonic stem cells into cardiomyocytes. Biochem Biophys Res Commun. 2006;342(1):107-12.

17. Shin DM, Ahn Jl, Lee KH, Lee YS, Lee YS. Ascorbic acid responsive genes during neuronal differentiation of embryonic stem cells. Neuroreport. 2004; 15(2):1959-63.

18. Ono M, Aratani Y, Kitagawa I, Kitagawa Y. Ascorbic acid phosphate stimulates type IV collagen synthesis and accelerates adipose conversion of 3T3-L1 cells. Exp Cell Res. 1990;187(2):309-14.

19. Kim B, Choi KM, Yim HS, Lee MG. Ascorbic acid enhances adipogenesis of 3T3-L1 murine preadipocyte through differential expression of collagens. Lipids Health Dis. 2013;12(1):182-9.

20. Weiser B, Sommer F, Neubauer M, Seitz A, Tessmar J, Goepferich A, et al. Ascorbic acid enhances adipogenesis of bone marrow-derived mesenchymal stromal cells. Cells Tissues Organs. 2009;189(6):373-81.

21. Choi KM, Yoon SHH. Effect of ascorbic acid on bone marrow-derived mesenchymal stem cell proliferation and differentiation. J Biosci Bioeng. 2008;105(6):586-94.

22. Zhang $Y$, Dong C. Regulatory mechanisms of mitogen-activated kinase signaling. Cell Mol Life Sci. 2007;64(21):2771-89.

23. Benito M, Porras A, Nebreda AR, Santos E. Differentiation of 3T3- L1 fibroblasts to adipocytes induced by transfection of ras oncogenes. Science. 1991:253(5019):565-8.

24. Aubert J, Belmonte N, Dani C. Role of pathways for signal transducers and activators of transcription, and mitogen-activated protein kinase in adipocyte differentiation. Cell Mol Life Sci. 1999;56(5-6):538-42.

25. Sale EM, Atkinson PG, Sale GJ. Requirement of MAP kinase for differentiation of fibroblasts to adipocytes, for insulin activation of p90 56 kinase and for insulin or serum stimulation of DNA synthesis. EMBO J. 1995;14(4):674-84

26. Janderová L, Mcneil M, Murrell AN, Mynatt RL, Smith SR. Human mesenchymal stem cells as an in vitro model for human adipogenesis. Obes Res. 2003;11(1):65-74.

27. Kim SW, Muise AM, Lyons PJ, Ro HS. Regulation of adipogenesis by a transcriptional repressor that modulates MAPK activation. J Biol Chem. 2001; 276(13):10199-206.

28. Yu J, YK T, Tang YB, Cheng NC. Stemness and transdifferentiation of adipose-derived stem cells using L-ascorbic acid 2-phosphate-induced cell sheet formation. Biomaterials. 2014;35(1):3516-26.

29. Park HJ, Sun MO, Kim HJ, Hong JP, Lee YB, Choi JM, et al. Vitamin C attenuates ERK signalling to inhibit the regulation of collagen production by LL-37 in human dermal fibroblasts. Exp Dermatol. 2010;19(8):E258-64.

30. Gong $M$, Liu C, Liang Z, Zhang $H$, Jie P. Loss of the TNFa function inhibits Wnt/ $\beta$-catenin signaling, exacerbates obesity development in adolescent spontaneous obese mice. Mol Cell Biochem. 2014;391(1):59-66.

31. Tang QQ, Otto TC, Lane MD. Mitotic clonal expansion: a synchronous process required for adipogenesis. Proc Natl Acad Sci U S A. 2003;100(1):44-9. 
32. Esteban MA, Wang T, Qin B, Yang J, Qin D, Cai J, et al. Vitamin C enhances the generation of mouse and human induced pluripotent stem cells. Cell Stem Cell. 2010;6(1):71-9.

33. Hayashida T, MH W, Pierce A, Poncelet AC, Varga J, Schnaper HW. MAPkinase activity necessary for TGFbeta1-stimulated mesangial cell type I collagen expression requires adhesion-dependent phosphorylation of FAK tyrosine 397. J Cell Sci. 2007;120(23):4230-40.

34. Varadharaj S, Steinhour E, Hunter MG, Watkins T, Baran CP, Magalang U, et al. Vitamin C-induced activation of phospholipase D in lung microvascular endothelial cells: regulation by MAP kinases. Cell Signal. 2006;18(9):1396-407.

35. Ulrichmerzenich G, Zeitler H, Panek D, Bokemeyer D, Vetter H. Vitamin C promotes human endothelial cell growth via the ERK-signaling pathway. Eur J Nutr. 2007:46(2):87-94.

36. Czuwaraladykowska J, Sementchenko VI, Watson DK, Trojanowska M. Ets1 is an effector of the transforming growth factor beta (TGF-beta ) signaling pathway and an antagonist of the profibrotic effects of TGF-beta. J Biol Chem. 2002:277(23):20399-408.

37. Mizui M, Isaka Y, Takabatake $Y$, Sato $Y$, Kawachi H, Shimizu F, et al. Transcription factor Ets-1 is essential for mesangial matrix remodeling. Kidney Int. 2006;70(2):298-305.

38. Bost F, Aouadi M, Caron L. Even P, Belmonte N, Prot M, et al. the extracellular signal-regulated kinase isoform ERK1 is specifically required for in vitro and in vivo adipogenesis. Diabetes. 2005;54(2):402-11.

39. Tang QQ, Otto TC, Lane MD. CCAAT/enhancer-binding protein $\beta$ is required for mitotic clonal expansion during adipogenesis. Proc Natl Acad Sci U S A. 2003:100(3):850-5.

40. Yin R, Mao SQ, Zhao B, Chong Z, Yang Y, Zhao C, et al. Ascorbic acid enhances Tet-mediated 5-methylcytosine oxidation and promotes DNA demethylation in mammals. J Am Chem Soc. 2013;135:10396-403.

41. Fujiki K, Shinoda A, Kano F, Sato R, Shirahige K, Murata M. PPARgammainduced PARylation promotes local DNA demethylation by production of 5hydroxymethylcytosine. Nat Commun. 2013;4:2262-70.

\section{Submit your next manuscript to BioMed Central and we will help you at every step:}

- We accept pre-submission inquiries

- Our selector tool helps you to find the most relevant journal

- We provide round the clock customer support

- Convenient online submission

- Thorough peer review

- Inclusion in PubMed and all major indexing services

- Maximum visibility for your research

Submit your manuscript at www.biomedcentral.com/submit 